\title{
Zero-degree algorithm for internet GateWay placement in backbone wireless mesh networks
}

\begin{abstract}
Internet GateWays (IGWs) are responsible for connecting the backbone wireless mesh networks (BWMNs) to the Internet/wired backbone. An IGW has more capabilities than a simple wireless mesh router (WMR) but is more expensive. Strategically placing the IGWs in a BWMN is critical to the Wireless Mesh Network (WMN) architecture. In order to solve the problem of IGWs placement in BWMNs, a novel algorithm is proposed in this paper. The new algorithm is involved in placing a minimum number of IGWs so that the Quality of Service (QoS) requirements are satisfied. Different from existing algorithms, this new algorithm incrementally identifies IGWs and prioritively assigns wireless mesh routers (WMRs) based on the computed degree of WMRs to identified IGWs. Performance evaluation results show that proposed algorithm outperforms other alternative algorithms by comparing the number of gateways placed in different scenarios. Furthermore, having control of the distribution of IGWs in order to locate them closest to available Internet/wired network connection points is an added advantage of this algorithm.
\end{abstract}

Keyword: Wireless mesh networks; Gateway placement; Optimization; Network Architecture; Network design 